\title{
Clinical Features and Outcome of Thyroid Storm: A Retrospective Study
}

\section{García ML1, Szuman G,, ${ }^{1}$ Scioscia MF1, Bacigaluppi S 1, Panebianco MV²,Rella N1.}

1 Sanatorio Dr Julio Méndez ,Buenos Aires. Argentina ${ }^{2}$ CEBBAD, Universidad Maimonides, Buenos Aires. Argentina

\section{OBJECTIVES}

To describe the clinical features and outcome of patients admitted for TS according to Burch-Wartofsky s score (BW).

\section{METHOD}

A retrospective review of subjects admitted to a single hospital from 2009 to 2015 was conducted.

\section{RESULTS}

Fifteen cases were identified: 12 women, 3 men. Average age 49,8 +/-17,3.

Resulting in four cases of Thyroid Storm per 10,000 admissions.

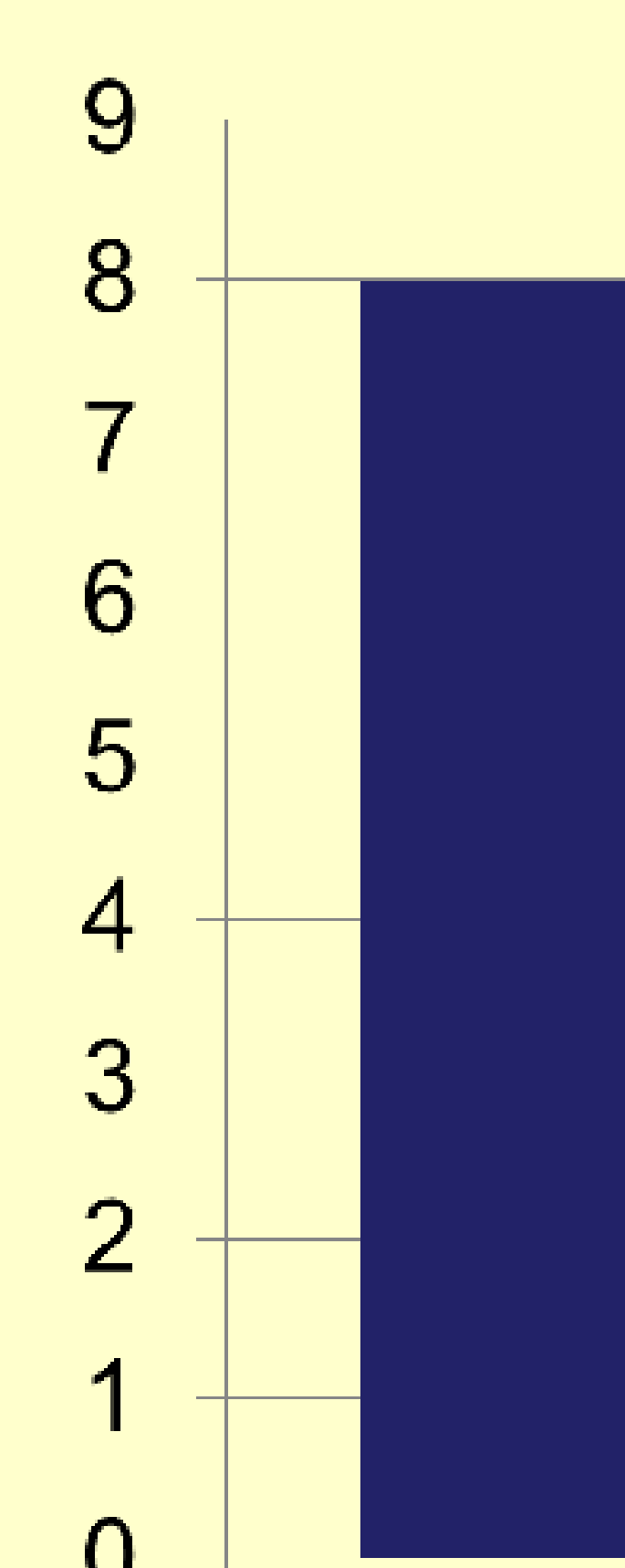

$c^{2}$
Reason for admission

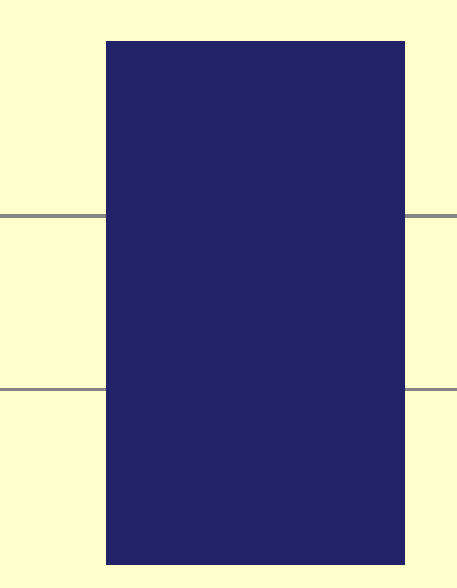<smiles>C=CC=C</smiles>

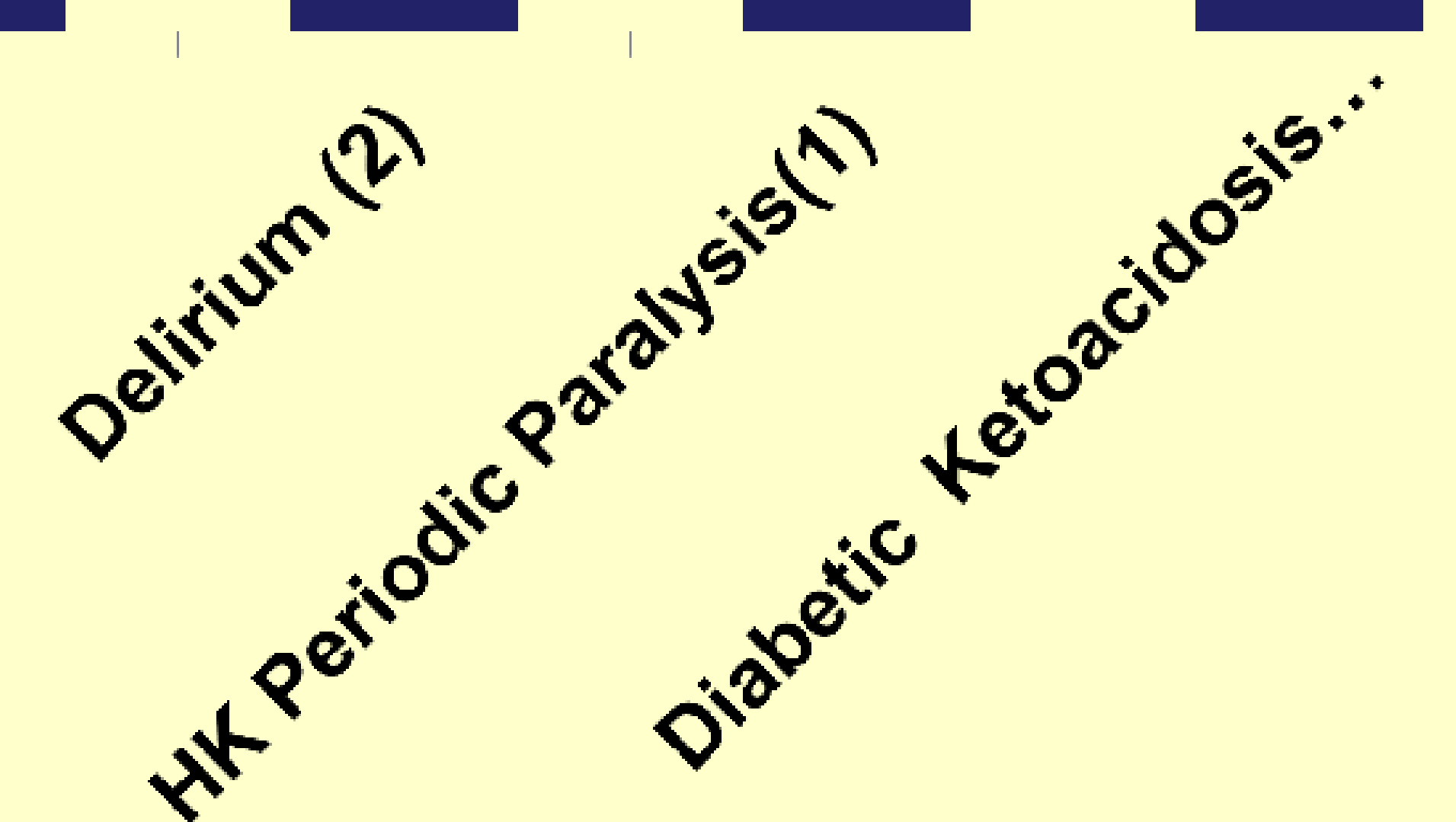

Hyperthyroidism Etiology

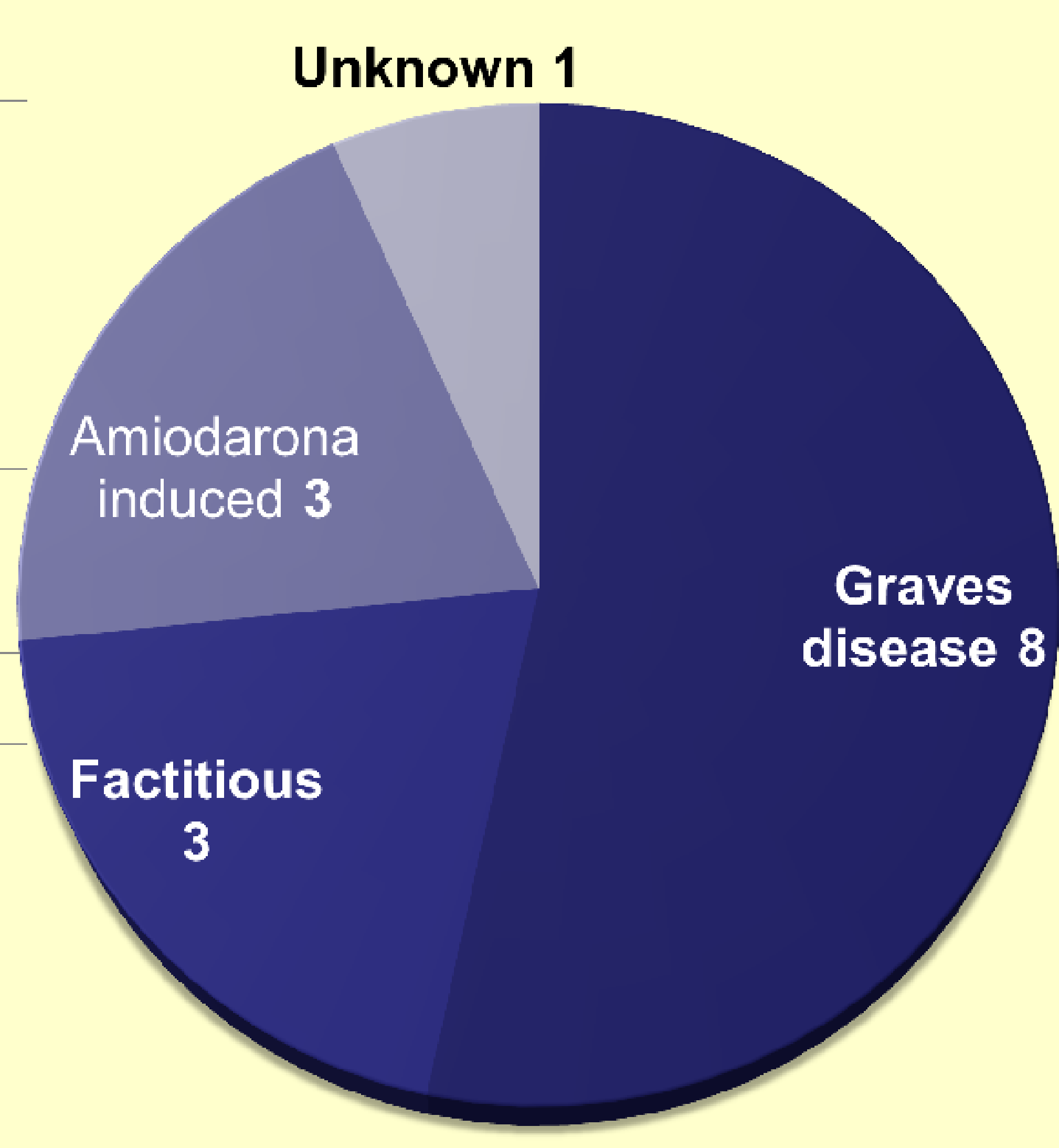

Precipitating Factors

Clinical manifestations according BW

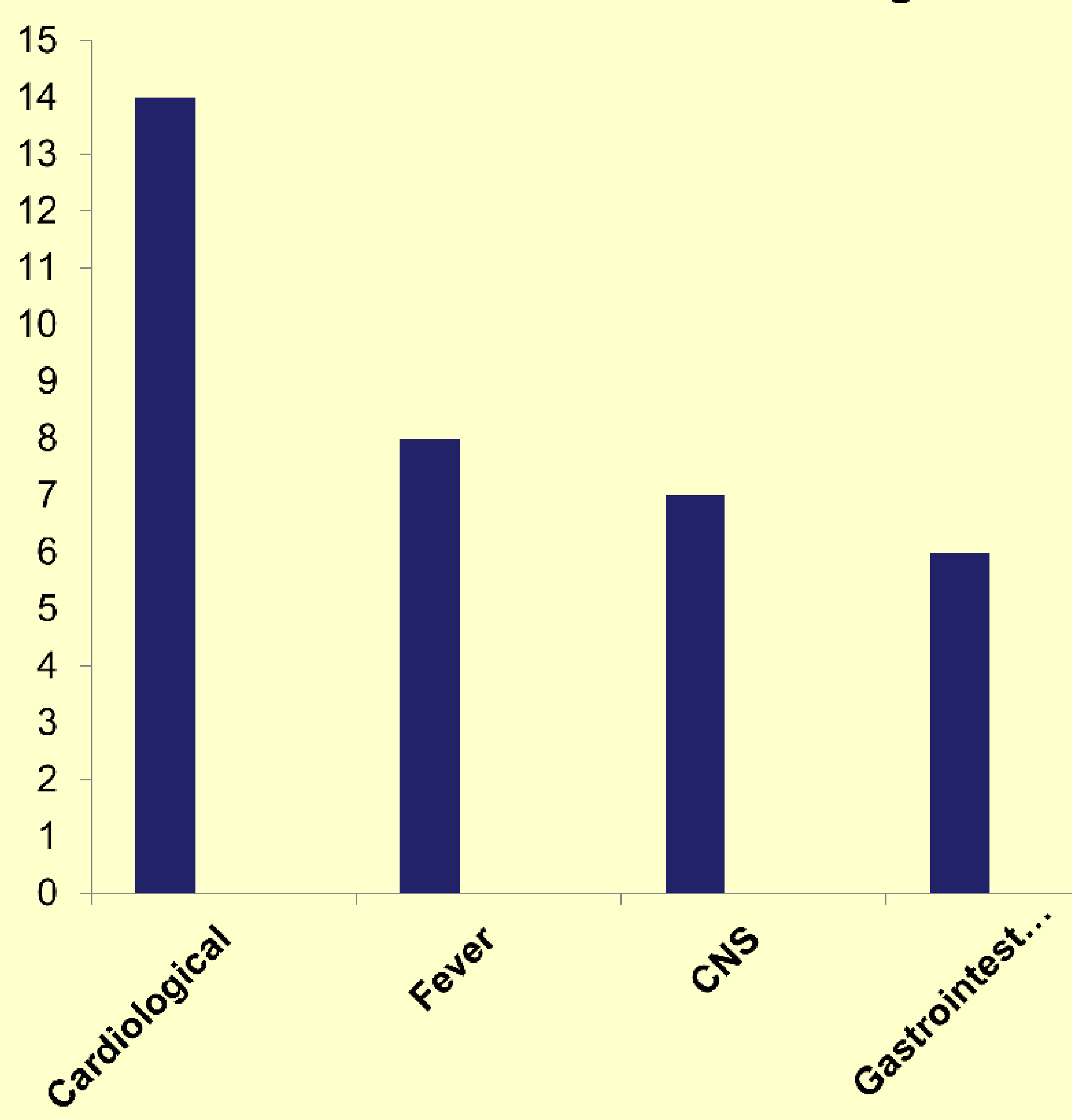

Precipitating Products
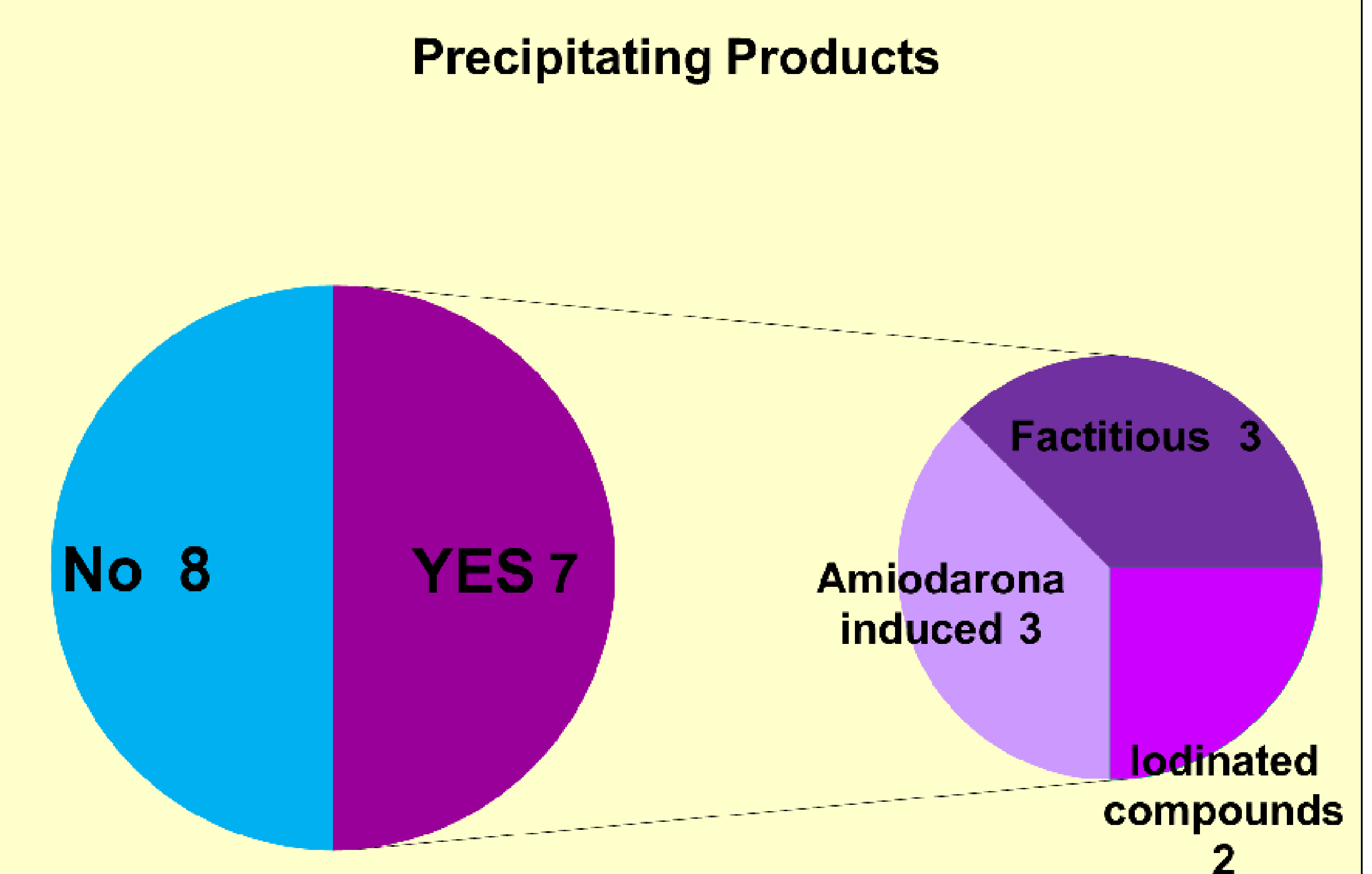

No previous thyroid disease (4)

Hypothyroidism (4)

- Hyperthyroidism (7)

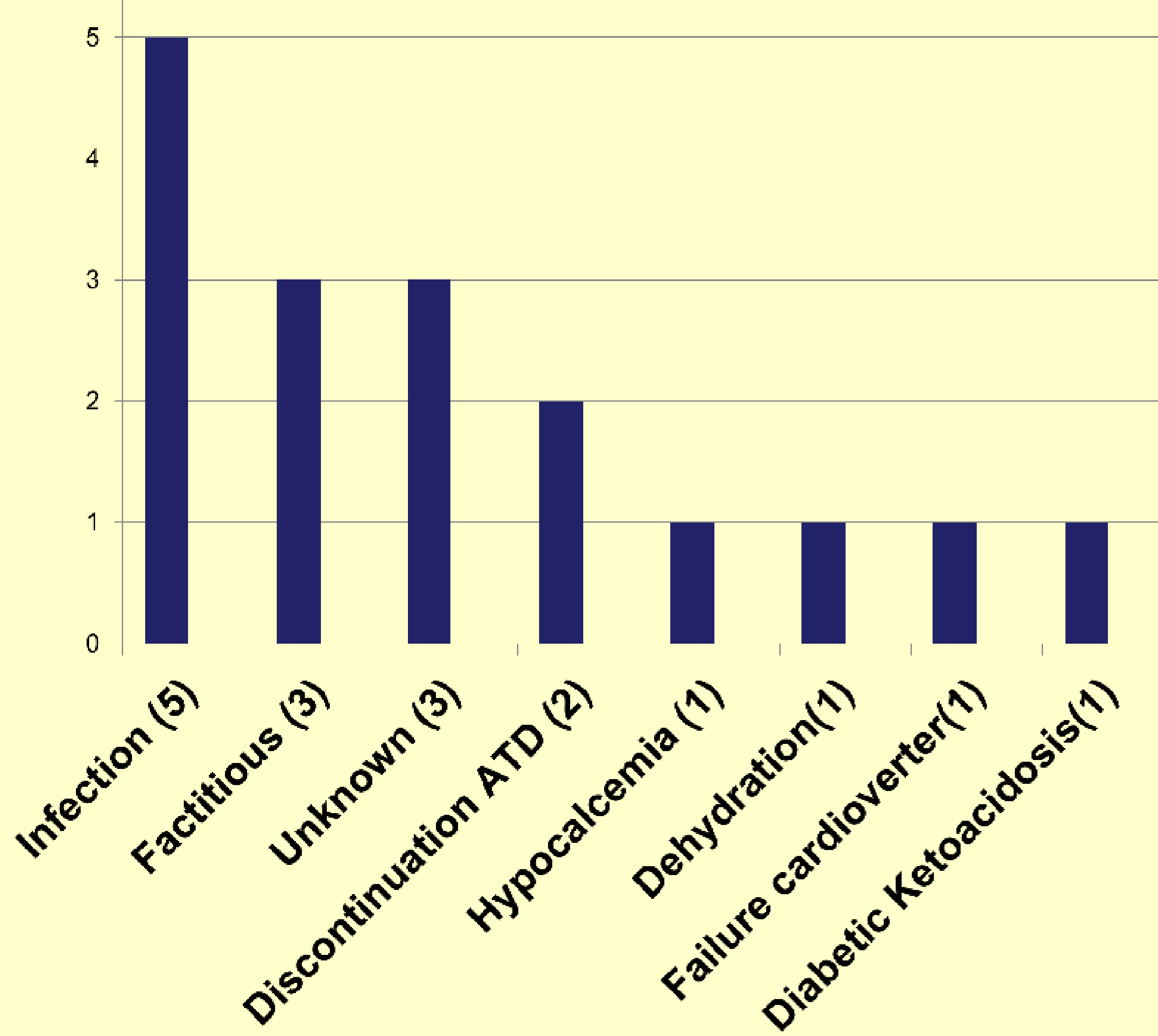

Hypokalemia (HK) was found in 8 cases, six of them probably due to the use of diuretics, one patient had hypokalemic periodic paralysis, one case due to treatment of diabetic ketoacidosis and one of unknown cause. Thyroid hormone levels did not correlate with the severity of thyrotoxicosis according to BW. One patient died from sepsis. Late hospital discharge was found in 4 patients: 3 required antithyroid drugs withdrawal (febrile neutropenia, cholestasis, digestive intolerance) and the other received a heart transplant.

\section{CONCLUSIONS}

The data collected in this series is not usually found in literature, and provides potentially useful information for the management of Thyroid Storm, a rare and acute disease that requires high clinical suspicion in order to improve patient $s$ survival. In this analysis, the most common etiology was autoimmune. Cardiovascular manifestations were predominant. Subjects with antithyroid drugs treatment had shorter time of hospitalization. Unusual findings for patients with thyroid storm, in this series were the high consumption of iodine and factitious thyrotoxicosis as precipitating factors, hypokalemia as a frequent laboratory finding, and the prior history of hypothyroidism 